\title{
RACIONALIZAÇÃO DAS AÇÕES DE CONTROLE INTERNO E AUDITORIA COMO PROCESSO DE CONTROLE NA GESTÃO DA SAÚDE EM UNIDADES HOSPITALARES NO CONTEXTO BRASILEIRO
}

\section{RATIONALIZATION OF INTERNAL CONTROL AND AUDITING ACTIONS AS A HEALTH MANAGEMENT CONTROL PROCESS IN HOSPITAL UNITS IN THE BRAZILIAN CONTEXT}

\section{RACIONALIZACIÓN DE LAS ACCIONES DE CONTROL INTERNO Y AUDITORÍA COMO PROCESO DE CONTROL EN LA GESTIÓN DE LA SALUD EN UNIDADES HOSPITALARES EN EL CONTEXTO BRASILEÑO}

\author{
Isabelle Christine GUERINI ${ }^{1}$ \\ Eduardo GUERINI ${ }^{2}$
}

\begin{abstract}
RESUMO: O presente artigo tem como objetivo descrever sucintamente a racionalização das ações de controle interno e auditoria nas gerências das unidades hospitalares no contexto brasileiro. A necessidade de mecanismos de gestão com implantação de auditagem derivou da necessidade de controles internos para melhoria contínua na gestão hospitalar, como resultado de um processo derivado da universalização proposta na regulação jurídica, garantindo a universalização do acesso, destacando uma clara orientação para estabelecimento de metas quantitativas e qualitativas. Elaborou-se breve histórico da implantação e da implementação de práticas gerenciais no sistema de saúde brasileiro, a racionalização, resultante de políticas públicas que são monitoradas por padrões de desempenho com resultados vinculados ao planejamento e cumprimento de metas almejadas. Neste sentido, observou-se que, no sistema de saúde pública ou privada, as variadas formas de organização hospitalar têm como claro resultado a transparência dos processos - elementos preponderantes para efetivação da prática de auditagem interna e externa nas unidades hospitalares no contexto brasileiro.
\end{abstract}

PALAVRAS-CHAVE: Racionalização; Unidades Hospitalares; Auditoria Interna e Externa; Sistema de Saúde.

Licença CC BY: Artigo distribuído sob os termos Creative Commons, permite uso e distribuição irrestrita em qualquer meio desde que o autor credite a fonte original
ABSTRACT This article aims to briefly describe the rationalization of control and internal audit in the management of hospital units in the Brazilian context. The need

1 Graduada em Enfermagem pela Universidade Federal de Santa Catarina - UFSC. Especialista em Auditoria Hospitalar.

2 Mestre em Sociologia Política pela Universidade Federal de Santa Catarina - UFSC. Graduado em Ciências Econômicas pela UFSC. Professor Universitário. | E-mail eduardo.guerini@univali.br. 
for management mechanisms to implement auditing stems from the need for internal controls for continuous improvement in hospital management. It is the result of a process that stems from the proposed universal legal regulation, ensuring universal access, highlighting a clear orientation towards the establishment of quantitative and qualitative targets. A brief historical of the introduction and implementation of management practices in the Brazilian health system, the rationalization that is the result of public policies that are monitored by performance standards results related to planning and meeting desired goals. In this regard, it was noted that both in the public health system and in the private one, the varied forms of hospital organization have a clear result, which is - transparency of the processes - predominant elements for the effective practice of internal and external auditing in hospitals in the Brazilian context.

KEY-WORDS: Rationalization; Hospitals; Internal and External Audit; Health System.

RESUMEN: El presente artículo tiene como objetivo describir sucintamente la racionalización de las acciones de control interno y auditoría en las gerencias de las unidades hospitalarias en el contexto brasileño. La necesidad de mecanismos de gestión con implantación de auditoría derivó de la necesidad de controles internos para mejoría continua en la gestión hospitalar, como resultado de un proceso derivado de la universalización propuesta en la regulación jurídica, garantizando la universalización del acceso, destacando una clara orientación para el establecimiento de metas cuantitativas y cualitativas. Se elaboró un breve histórico de la implantación y la implementación de prácticas gerenciales en el sistema de salud brasileño, la racionalización, resultante de políticas públicas que son monitoreadas por padrones de desempeño con resultados vinculados a la planificación y cumplimiento de metas buscadas. En este sentido, se observó que, en el sistema de salud pública o privada, las variadas formas de organización hospitalar tienen como claro resultado la transparencia de los procesos - elementos preponderantes para efectuación de la práctica de auditoría interna y externa en las unidades hospitalarias en el contexto brasileño.

PALABRAS-CLAVE: Racionalización; Unidades Hospitalarias; Auditoría Interna y Externa; Sistema de Salud.

\section{INTRODUÇÃO}

No Brasil, as políticas públicas de saúde são definidas pelo Ministério da Saúde (MS), o qual estabelece as metas assistenciais, de ensino e de pesquisa, de humanização e de gestão. Numa visão geral, as políticas públicas de saúde são influenciadas pelo Estado e afetam a sociedade. O Estado assume a responsabilidade por uma política de saúde integrada às demais políticas sociais e econômicas. O Conselho Nacional de Saúde (CNS) estabelece as diretrizes a serem observadas na elaboração dos planos de saúde, de acordo com as características epidemiológicas e da organização de serviços nos entes federativos e regionais de saúde. O CNS tem por finalidade, de acordo com a Resolução 407 de 12 de setembro 2008, "atuar na formulação e no controle da execução da Política Nacional de Saúde, inclusive nos aspectos econômicos e financeiros, nas estratégias e na promoção do processo de controle social em toda a sua amplitude.” (BRASIL, 2008).

Para garantir o acesso universal à saúde para toda a população, a Constituição Federal (CF) de 1988 cria o Sistema Único de Saúde (SUS) e estabelece no Art. 196 “A saúde é direito de todos e dever do Estado, garantido mediante políticas sociais e econômicas que visam à redução do risco de doença e de outros agravos e ao acesso universal e igualitário às ações e serviços para sua promoção, proteção e recuperação” (BRASIL, 1988). A Lei 8080, de 19 de setembro de 1990, constitui efetivamente o Sistema Único de Saúde (SUS) como "O conjunto de ações e serviços de saúde, prestados por órgãos e instituições públicas federais, estaduais e municipais, 
da administração direta e indireta e das fundações mantidas pelo Poder Público.” (BRASIL, 1990). Esta lei também consolida os princípios de sua operacionalização: um sistema de saúde descentralizado, regionalizado e hierarquizado; e os princípios doutrinários: a universalidade, a integralidade e a igualdade nos serviços e nas ações de saúde.

Os Ministérios da Educação e da Saúde estabelecem o Programa de Reestruturação dos Hospitais de Ensino (REHUF), por meio da Portaria Interministerial $\mathrm{n}^{\circ}$ 1006/MEC/MS de 27 de maio de 2004, para atender as necessidades de reorientação e reformulação das políticas de saúde do SUS para os hospitais de ensino. O Programa objetiva as ações estratégicas fundamentadas nos princípios e nas diretrizes do SUS mediante processo de contratualização.

O processo de contratualização dos hospitais é uma reformulação da política de atenção à saúde a partir da pactuação de regras entre o representante legal do hospital e o gestor do SUS. As metas quantitativas e qualitativas do processo de atenção à saúde da gestão hospitalar são determinantes na racionalização do processo decisório nas unidades de saúde. Os processos de acompanhamento são avaliados mensalmente, com adoção de relatórios de desempenho que tornam a gestão mais eficiente e efetiva de acordo com as diretrizes do SUS, na atenção e na liberação de recursos financeiros às unidades hospitalares.

A integralização das unidades hospitalares na rede regionalizada tem por finalidade a efetividade das políticas de saúde do sistema de universalização da saúde no Brasil.

A rede pública de serviços de saúde é referência em tratamentos clínicos e cirúrgicos, em caráter preventivo, curativo e reabilitador a pacientes em regime de atendimento ambulatorial e internação. Os hospitais são organizações de alta complexidade, apresentam uma equipe multidisciplinar com elevado grau de autonomia para dar assistência à saúde; são locais de prática de ensino, aprendizagem e produção científica para atender a necessidade da população na área da saúde.

As prestações de serviços de saúde exigem um atendimento de qualidade e segurança garantida aos usuários em busca dos resultados satisfatórios na evolução e na cura das enfermidades apresentadas. A qualidade nos serviços de saúde inclui não só atendimento assistencial, mas também nas ações de controle interno para atender as metas pactuadas e cumprir com os objetivos das políticas sociais do Estado.

Visando fortalecer a gestão e racionalizar as ações de controle, o Presidente da República, Fernando Henrique Cardoso, determina em 2002 que as entidades da Administração Pública Federal Indireta implantem a unidade de auditoria interna, com o objetivo de promover as ações de controle, conforme: art. 15, § 60 do Decreto 4304, de 16 de julho de 2002, “A auditoria interna examinará e emitirá parecer sobre a prestação de contas anual da entidade e tomadas de contas especiais”; art. 14 do Decreto 4440, de 25 de outubro de 2002, "As entidades da Administração Pública Federal indireta deverão organizar a respectiva unidade de auditoria interna, com o suporte necessário de recursos humanos e materiais, com o objetivo de fortalecer a gestão e racionalizar as ações de controle."

Avaliar a qualidade nos serviços de saúde é necessário para o alcance dos objetivos esperados. A alta administração necessita de monitoramento, avaliação e adequação permanente dos controles internos em busca da qualidade nos serviços de saúde. Segundo Melamed e Piola (2011, p.198), "Mesmo com o aperfeiçoamento do sistema de críticas pelo SUS, a qualidade das informações constitui um dos principais problemas a ser enfrentados...”. 
Para Ruthes e Cunha (2007, p.94), “o setor de saúde no Brasil e no mundo é alvo de investimentos financeiros crescentes, daí a exigência de maior eficiência e qualificação de pessoas”. O atendimento hospitalar possui alto custo pelos avanços tecnológicos tanto do ponto de vista diagnóstico, com novos exames, quanto do ponto de vista terapêutico, com novos procedimentos e medicamentos. Os hospitais universitários federais são penalizados quando não cumprem as metas quantitativas e qualitativas pactuadas. Para atender os serviços de saúde, inclui não só atendimento assistencial, mas também ações de controle interno para o alcance dos resultados previstos na contratualização com o gestor.

A falta de uma auditoria interna para avaliar, controlar e adequar os controles internos compromete a qualidade dos serviços de saúde e a efetividade das políticas do SUS. De acordo com a opinião do diretor do Centro de Relações Internacionais em Saúde da Fundação Oswaldo Cruz - Fiocruz:

A solução para os problemas na área de saúde - tanto no Brasil como nos demais países - não é apenas uma questão de se aplicar mais dinheiro. É preciso se investir em administração, para que os recursos disponibilizados cheguem na ponta do sistema, sem se perder no meio do caminho, por corrupção ou má gestão. (BUSS, 2011).

Considerando os problemas nas ações de controle interno nas unidades hospitalares, a auditoria interna pode auxiliar no processo de racionalização e no processo decisório de gestão de unidades de saúde.

A auditoria interna no processo de racionalização das ações de controle interno no serviço de saúde busca efetivar uma gestão racional das políticas públicas de saúde de forma integrada e articulada entre os diversos agentes operadores do sistema de saúde no Brasil.

Uma auditoria interna para o processo de racionalização nas ações de controle é importante instrumento para o alcance da economicidade, eficiência, eficácia, efetividade e equidade do desempenho organizacional nas unidades hospitalares. Segundo Gurgel Jr. e Vieira (2002, p.1), “o alcance da qualidade nos serviços de saúde não está diretamente sobre o ato clínico, mas nos processos administrativos da organização hospitalar, nas áreas da organização que garantem o funcionamento dos setores operacionais.”

Propor uma auditoria interna nas Unidades de Saúde para o processo de racionalização das ações nos controles operacionais tem por finalidade: obter a qualidade dos registros dos procedimentos no Sistema Interno Hospitalar (SIH); reduzir as perdas de recursos financeiros por procedimentos não registrados; elaborar um fluxo dos processos de trabalho; atender os auditores externos sem ressalvas no âmbito da instituição, contribuindo para o princípio da boa governança; e auxiliar a gestão para a tomada de decisão.

O estudo de caso nas Unidades Hospitalares tem como justificativa fundamentar os benefícios da prática das atividades de uma auditoria interna, como: propor correção de ações inadequadas por mais eficientes, reduzir as não conformidades nos processos de faturamento, prevenir irregularidades e auxiliar a gestão na qualidade da sua operacionalização. 


\section{POLÍTICAS PÚBLICAS DE SAÚDE E NOVAS FORMAS DE GESTÃO NO CONTEXTO BRASILEIRO}

A política pública surgiu nos EUA da área de conhecimento e disciplina acadêmica e, na Europa, como um desdobramento dos trabalhos baseados em teorias explicativas sobre o papel do Estado. A definição mais conhecida, para Souza (2006, p. 24), "continua sendo a de Laswell, ou seja, decisões e análises sobre política pública implicam responder às seguintes questões: quem ganha o quê, por quê e que diferença faz”.

Para Secchi (2010, p.2), “qualquer definição de políticas públicas é arbitrária”. A política pública tem como meta resolver os problemas do cidadão: “A política pública é um exercício do setor público, o qual devolve à população as contribuições que ela realiza pagando impostos, alíquotas, taxas e tarifas. Os retornos são soluções de problemas sociais, econômicos, ambientais, entre outros" (SILVA; SOUZA-LIMA, 2010).

Nas últimas décadas, três fatores que contribuíram para maior importância na área das políticas públicas: a adoção de políticas restritivas aos gastos, em especial nos países em desenvolvimento; o ajuste fiscal que implicou os orçamentos equilibrados entre as receitas e as despesas, a necessidade de impulsionar o desenvolvimento econômico e de promover a inclusão social.

Os principais fundadores desta área, segundo Souza (2006), são H. Laswell (1936), que introduz a expressão policy analysis; H. Simon (1957), que introduz o conceito policy makers; C. Lindblom (1959; 1979), que propôs a incorporação de outras variáveis à formulação e à análise de políticas públicas; e D. Easton (1965), que contribuiu ao definir a política pública como um sistema composto de formulação, resultados e ambiente.

As políticas públicas são formuladas por grupos de interesse, o Estado e a sociedade, os quais identificam os problemas e definem os programas das políticas públicas. O objetivo primordial das políticas públicas é solucionar os problemas que foram definidos. Identificados os principais problemas, são contemplados nas políticas públicas em busca de soluções. Depois de colocadas em ação, são avaliadas e analisadas, e quando necessário sofrem reformulações.

De um modo geral, as políticas necessitam de envolvimento e planejamento estratégico, segundo Rua (2009, p.19), "política pública envolve mais que uma decisão e requer diversas ações estrategicamente selecionadas para implementar as decisões tomadas.” Investigados os principais problemas, são contemplados nas políticas públicas em busca de soluções. Segundo Souza (2006, p.40),

[...] o principal foco analítico da política pública está na identificação do tipo de problema que a política pública visa corrigir, na chegada desse problema ao sistema político (politics) e à sociedade política (polity), e nas instituições/ regras que irão modelar a decisão e a implementação da política pública.

O controle das políticas é composto por quatro fases distintas: estabelecimento de padrões de desempenho; mensuração do desempenho: comparação do desempenho atual com o padrão; e tomada de ação corretiva para ajustar o desempenho atual ao padrão desejado. Se for um caso de ajuste, pode ser proposta implementação. Para Rua (2009, p.94), “a implementação, portanto, 
compreende o conjunto dos eventos e atividades que acontecem após a identificação das diretrizes de uma política, incluem tanto o esforço para administrá-la, como seus substantivos sobre pessoas e eventos.”.

O processo de elaboração de políticas públicas, segundo Secchi(2010, p.33), “... também é conhecido como ciclo de políticas públicas (policy cycle).”. O ciclo favorece a visualização e o entendimento das fases sequenciais e interdependentes do processo de elaboração. Segundo Rua (2009, p.37), “o ciclo de políticas é uma abordagem para o estudo das políticas públicas que identifica fases sequenciais e interativas-iterativas no processo de produção de uma política.” O ciclo das políticas públicas, segundo Secchi (2010, p.33), restringe “...o modelo às setes fases principais: 1) identificação do problema, 2) formação da agenda, 3) formulação de alternativas, 4) tomada de decisões, 5) implementação, 6) avaliação, 7) extinção.”.

O problema de saúde exige que o Estado assuma a responsabilidade por uma política de saúde integrada às demais políticas sociais e econômicas e garanta a sua efetivação. A política deve percorrer o conjunto das ações e dos projetos em saúde, apresentando-se em todos os níveis de complexidade da gestão e da atenção do sistema de saúde. De acordo com Campos, Barros e Castro (2004, p.2), "uma política, portanto, comprometida com serviços e ações de saúde que coloquem os sujeitos - usuários e profissionais de saúde - como protagonistas na organização do processo produtivo em saúde, entendendo que aí se produz saúde”. O Conselho Nacional de Saúde estabelece as diretrizes a serem observadas na elaboração dos planos de saúde, de acordo com as características epidemiológicas e da organização de serviços nos entes federativos e nas regiões de saúde. Para uma política Nacional de Promoção da Saúde que seja efetiva e eficaz para a melhoria da qualidade de vida da população são necessários compromissos éticos da política e do sistema de saúde brasileiros. A utilização do Mapa da Saúde contribui para a identificação das necessidades de saúde e orientação para o estabelecimento das metas de saúde.

O processo de planejamento da saúde é ascendente e integrado, do nível Regional até o Federal, compatibilizando-se às necessidades das políticas de saúde com a disponibilidade de recursos financeiros. Segundo Campos, Barros e Castro (2004), a política nacional de promoção da saúde apresenta-se em todos os níveis de complexidade da gestão e da atenção do sistema de saúde. É um campo teórico prático-político com os conceitos e delineia-se como uma política que deve percorrer o conjunto das ações e dos projetos em saúde.

Segundo Carvalho e Barbosa (2010), a partir de 1945, com o aumento progressivo da demanda por atenção à saúde, foi promulgada a Lei da Previdência Social (LOPS) em 1960, que estabeleceu a padronização dos serviços de saúde, unificou os benefícios a todos os segurados, independente do instituto a que estivessem filiados. O aumento da população e da prestação de serviços contribuiu para a concentração da rede da Previdência Social nas grandes cidades, porém o cidadão não previdenciário continuou discriminado.

Com a criação do Instituto de Previdência Social (INPS) em 1966, ocorre a unificação de todas as instituições previdenciárias setoriais. A saúde previdenciária passou a ser substituída pelos serviços hospitalares próprios da Previdência Social. Por falta de planejamento, controle e alto custo não teve bons resultados.

A Lei 6.229, de 17 de julho de 1975, estabelece o Sistema Nacional de Saúde, visando corrigir os 
problemas no campo das ações de saúde. Foi atribuído ao Ministério da Saúde a formulação da política de saúde. Mais uma vez o sistema de saúde não alcançou o resultado esperado.

A criação do Programa de Desenvolvimento de Sistema Unificados e Descentralizados de Saúde (SUDS), por meio do Decreto 94657 de 20 de julho de 1987, redefiniu as funções e as atribuições dos Estados e dos Municípios no campo da saúde. O programa estabeleceu a descentralização de recursos humanos e financeiros aos Estados e aos Municípios, ficando a União como coordenadora, com as atribuições de planejar, financiar e acompanhar as políticas de saúde.

O Ministério da Saúde estabelece a Comissão de Avaliação da Atenção Básica, constituída pela Portaria GM/MS N 676 de 03/06/03, para acompanhar e avaliar as políticas públicas de saúde. Segundo Felisberto (2006, p. 553):

O Ministério da Saúde, por intermédio da Coordenação de Acompanhamento e Avaliação do Departamento de Atenção Básica, vem, a partir de 2003, desenvolvendo diversas ações com vistas à implantação de uma política de avaliação para a atenção básica, calcada em algumas diretrizes: 1) Parcerias com instituições de ensino e pesquisa nacionais e internacionais, bem como com associações profissionais de reconhecido prestígio político e acadêmico; 2) Mobilização de atores institucionais por intermédio de processos amplamente participativos, que buscaram não apenas o estabelecimento de alianças no desenvolvimento de projetos, mas que permitiram, sobretudo, a adesão e incorporação de propostas de forma mais coerente com as múltiplas necessidades do sistema de saúde; 3) Identificação de fontes de financiamento e garantia de recursos orçamentários para os projetos; 4) Definição de estratégias organizacionais para qualificação de profissionais no campo da avaliação.

Na ação do governo para reduzir a desigualdade social e garantir a justiça social, foi estabelecido o Sistema Único de Saúde (SUS), por meio da Constituição Federal (CF) de 1988. O SUS prevê o acesso universal e igualitário às ações e aos serviços de saúde. Para atender estas ações, surgem as políticas públicas de saúde, as quais são definidas pelo Ministério da Saúde. A CF de 1988 estabelece no Art. 196: "A saúde é direito de todos e dever do Estado, garantido mediante políticas sociais e econômicas que visam à redução do risco de doença e de outros agravos e ao acesso universal e igualitário às ações e serviços para sua promoção, proteção e recuperação”. Considerando este artigo da $\mathrm{CF}$, as políticas sociais visam à justiça social e repercutem na economia e na sociedade.

\section{CARACTERÍSTICAS DA UNIVERSALIZAÇÃO DOS SISTEMA DE SAÚDE NO BRASIL}

O Sistema Único de Saúde é um sistema público para oferecer atendimento a todas as pessoas, em todo o território nacional, sob a responsabilidade das três esferas do Governo Federal, Estadual e Municipal.

Uma das características do SUS é a descentralização da gestão, com Comissões Intergestores Tripartites, transferência de recursos e atribuições da União, Estados e Municípios. O Ministério da Saúde, como órgão definidor das políticas do SUS, promove, articula e integra atividades e ações de cooperação técnica. 
O Sistema Único de Saúde (SUS) constitui um moderno modelo de organização dos serviços de saúde e tem como uma das suas características primordiais fornecer cobertura integral de saúde a todos os cidadãos do país. Para promover a saúde, o novo modelo prevê as ações preventivas que visam à redução do risco de doenças. Segundo Carvalho e Barbosa (2010, p. 39):

O novo desenho político-institucional faz referência à ideia de que a implantação do novo sistema de saúde, agora universal, deveria se dar de forma inovadora, com estruturas ágeis e compatíveis com as novas tarefas e os novos compromissos do sistema.

O novo modelo técnico-assistencial é baseado em uma concepção ampliada do processo saúde-doença, em que o acesso a bens e aos serviços de saúde é apenas um dos componentes de um processo que depende essencialmente de políticas públicas mais amplas, capazes de promover qualidade de vida.

A Lei 8080, de 19 de setembro de 1990, constitui efetivamente o Sistema Único de Saúde-SUS como "O conjunto de ações e serviços de saúde, prestados por orgãos e instituições públicas federais, estaduais e municipais, da administração direta e indireta e das fundações mantidas pelo Poder Público.” (BRASIL, 1990). Esta lei estabelece a consolidação dos princípios de sua operacionalização: um sistema de saúde descentralizado, regionalizado e hierarquizado; e os princípios doutrinários: a universalidade, a integralidade e a igualdade nos serviços e nas ações de saúde.

A Lei 8.080, de 19 de setembro de 1990, dispõe sobre as condições para a promoção, proteção e recuperação da saúde, a organização e o funcionamento dos serviços de saúde. Também foram estabelecidos os princípios e as diretrizes do SUS:

Art. $7^{\circ}$ As ações e serviços públicos de saúde e os serviços privados contratados ou conveniados que integram o Sistema Único de Saúde - SUS são desenvolvidos de acordo com as diretrizes previstas no artigo 198 da Constituição Federal, obedecendo ainda aos seguintes princípios:

I - universalidade de acesso aos serviços de saúde em todos os níveis de assistência;

II - integralidade de assistência, entendida como um conjunto articulado e contínuo das ações e serviços preventivos e curativos, individuais e coletivos, exigidos para cada caso em todos os níveis de complexidade do sistema;

III - preservação da autonomia das pessoas na defesa de sua integridade física e moral;

IV - igualdade da assistência à saúde, sem preconceitos ou privilégios de qualquer espécie;

V - direito à informação, às pessoas assistidas, sobre sua saúde;

VI - divulgação de informações quanto ao potencial dos serviços de saúde e sua utilização pelo usuário;

VII - utilização da epidemiologia para o estabelecimento de prioridades, a alocação de recursos e a orientação programática; 
VIII - participação da comunidade;

IX - descentralização político-administrativa, com direção única em cada esfera de governo:

a) ênfase na descentralização dos serviços para os municípios;

b) regionalização e hierarquização da rede de serviços de saúde;

$\mathrm{X}$ - integração, em nível executivo, das ações de saúde, meio ambiente e saneamento básico;

XI - conjugação dos recursos financeiros, tecnológicos, materiais e humanos da União, dos Estados, do Distrito Federal e dos Municípios na prestação de serviços de assistência à saúde da população;

XII - capacidade de resolução dos serviços em todos os níveis de assistência; e

XIII - organização dos serviços públicos de modo a evitar duplicidade de meios para fins idênticos. (BRASIL, 1999).

\section{A ORGANIZAÇÃO HOSPITALAR E A RACIONALIZAÇÃO DOS SERVIÇOS DE SAÚDE}

A organização hospitalar é uma das mais complexas e requer alta qualidade nos serviços de saúde, não apenas pela nobreza e amplitude da sua missão, mas, sobretudo, por apresentar uma equipe multidisciplinar com elevado grau de autonomia, para dar assistência em caráter preventivo, curativo e realibilitador a pacientes em regime de internação, no qual se utiliza tecnologia de ponta. De acordo com Mintzberg (1995), a organização hospitalar do ponto de vista estrutural caracteriza-se por ser uma burocracia profissional, cujo setor operacional tem importância, traciona e concentra o poder na organização.

Segundo Drucker (1975 apud Mezzomo e Cherubin, 1986, p. 82), "O hospital é a mais complexa das empresas modernas.”. A complexidade dos hospitais revela-se em função dos serviços muito diversificados, desde o administrador que se relaciona com profissionais com doutorado a funções de apoio; na divisão e no uso do espaço físico, o mesmo espaço sendo ocupado por um grande fluxo de pessoas; no componente humano que há uma grande diversificação e carência de qualificação; nas exigências múltiplas de sua clientela, pacientes e familiares; nas urgências das ações; na escassez de seus serviços físicos, materiais, humanos e financeiros; na interligação e na interdependências dos serviços.

No caso das políticas sociais relacionadas aos serviços da saúde, os hospitais universitários federais são os agentes executores das políticas públicas de saúde. As avaliações e a análise das ações são fundamentais para a efetividade destas políticas públicas.

Os hospitais públicos e privados, como unidades de saúde para atendimento do público em geral, atualmente estão vinculados ao Ministério da Saúde no Brasil. Essas unidades desempenham as funções de centros de referência de média e alta complexidade e são consideradas locais de referência em tratamentos clínicos e cirúrgicos de alta complexidade, em caráter preventivo, curativo e reabilitador a pacientes em regime de atendimento ambulatorial e internação. 
Uma gestão de conhecimento em serviços de saúde, segundo Gonçalo e Borges (2010, p.4), "significa alinhar pessoas, processos, dados e tecnologias para otimizar informações, colaboração, especializações e experiências com habilidade para impactar positivamente a performance organizacional.”. A necessidade de manter transparências das ações com informações adequadas e fidedignas atende aos princípios de racionalização na gestão e atendem aos requisitos de auditagem externa e interna para uma gestão eficiente e efetiva nas ações de saúde dos agentes e dos pacientes no sistema de saúde no Brasil.

\section{AUDITORIA INTERNA EM UNIDADES HOSPITALARES COMO RACIONALIZAÇÃO DA GESTÃO}

A palavra auditoria originou do latim "audire”, que significa ouvir. Não se tem uma história precisa de quando iniciou a auditoria pelo fato que qualquer pessoa que prestasse contas a um ser superior era considerada um auditor. Na Inglaterra, o Rei Eduardo I, da dinastia Plantageneta, entre 1272 e 1307, mandou verificar as contas do testamento de sua falecida esposa. Os documentos foram aprovados com atestes, os quais constituíram os primeiros relatórios de auditoria, denominados "probatur sobre as contas".

A auditoria iniciou no Brasil pela necessidade de prestar contas e controlar os recursos financeiros. O primeiro parecer de auditoria foi publicado em 1903, relativo ao exame nos livros da São Paulo Tramway, Light and Power Company. A auditoria evoluiu por servir de assessoramento aos administradores. As organizações passaram a utilizar a auditoria interna para avaliar e adequar os controles internos, examinar os procedimentos administrativos e reduzir custos.

Os conceitos apresentados da auditoria interna tem por finalidade demonstrar a amplitude da sua aplicação e agregar um valor mais preciso nos resultados das organizações. Segundo Magalhães, Lunkes e Müller (2001, p.20), a auditoria interna tem como objetivo o "acompanhamento dos atos e registros da gestão, previsão de erros e/ou de fraudes, sugestões de políticas administrativas e estratégias para a organização.”. Para os autores, a auditoria sofreu mutações na sua lógica conceitual, passando a ter uma ampla visão de assessoramento à alta administração na tomada de decisão, no planejamento estratégico e na prevenção de riscos dentro e fora da área contábil.

As Normas Brasileiras de Contabilidade (NBC T - 12), aprovadas pelo Conselho Federal de Contabilidade (CFC), por meio da Resolução nº 986/03 de 21 de novembro de 2003, conceitua a auditoria interna como: "A auditoria interna constitui o conjunto de procedimentos técnicos que tem por objetivo examinar a integridade, adequação e eficácia dos controles internos e das informações físicas, contábeis, financeiras e operacionais da Entidade” (NBC T 12.1.1.1, 2001). O CFC conceitua na perspectiva de prestação contas e do controle dos recursos financeiros.

Castro (2008, p.157) afirma que a auditoria interna "consiste num mecanismo de controle, na medida em que revisa e avalia a eficiência e adequação dos outros controles existentes e auxilia a alta administração na tomada de decisão.”. O autor enfatiza em seu conceito o benefício de assessoramento da gestão administrativa.

Atualmente, a auditoria interna é vista de maneira diferente, passou a ter um enfoque de assessoramento administrativo, dentro e fora da área contábil. Tem como objetivo avaliar, 
acompanhar e propor adequação dos atos de gestão no âmbito da organização; exerce uma ação preventiva, moralizadora e saneadora; visa à eficiência, à eficácia, à efetividade e à equidade nas questões relativas à aplicação dos recursos, dos controles internos e da gestão.

Na gestão de unidades de saúde, hospitalares e ambulatoriais, o diagnóstico da auditagem deve condicionar os Registros do SIH, os Procedimentos realizados (RNM) no sistema de internação (SIH), as informações registradas no Tabwin (Sistema de Tabulação de Dados do Ministério da Saúde), informações dos arquivos de faturamento ambulatorial, Prontuários e Laudos de Exames de pacientes com AIHs geradas no SIH por período contabilizado, comparando com as notas fiscais pagas ao serviço contratado, relatórios de exames realizados, e, finalmente, o relatório de auditagem interna, com claro objetivo de controle racional da gestão eficiente da saúde.

O acompanhamento dos processos de trabalho e ações de controle atendem à racionalização do processo de trabalho que atende setores que anteriormente as reformas na gestão da saúde públicas, com diretrizes claras e objetivas com dispêndio de recursos, atendem em última instância, a aplicação adequada de fluxos financeiro-contábeis na gestão hospitalar.

A auditoria tende a sanear e sanar as distorções nos aspectos procedimentais, gerando clareza nas informações e regularizando as distorções no fluxo administrativo das ações de saúde em unidades hospitalares, permitindo o gerenciamento adequado dos recursos escassos e construindo uma nova gestão focada em resultados para constituição de boas práticas procedimentais na gestão das unidades de saúde.

A gestão adequada de informações, fluxos dos processos de trabalho, com a racionalização dos controles internos, contribuem para a redução dos custos operacionais das unidades de saúde, adequação nos sistemas de registro de exames no SIH, estabelecimento de padrões entre exames autorizados, realizados e faturados, otimizando as receitas dos serviços de saúde realizado.

Os benefícios almejados na implantação de um sistema de controle interno, via auditagem rotineira nos processos e nos procedimentos, tornaram-se imprescindíveis na gestão das unidades de atendimento hospitalar, com benefícios no âmbito institucional para melhoria da qualidade dos serviços prestados na área da saúde.

\section{CONSIDERAÇÕES FINAIS}

A universalização da saúde no contex to brasileiro, resultante da aprovação de um marco regulatório insculpido na Constituição de 1988, proporcionou a implementação de novos padrões na gestão de unidades hospitalares. Os aspectos gerenciais em termos de metas quantitativas e qualitativas na literatura da avaliação das políticas públicas da saúde, assim como na reforma gerencial do Estado brasileiro, ensejaram práticas distintivas em termos de efetividade, eficácia e eficiência, como métodos e técnicas de avaliação e monitoramento nas unidades hospitalares.

A constituição de uma nova agenda nas políticas públicas de saúde brasileira-via universalização do sistema único - se traduz em vetores da mudança no processo de gestão da saúde, seja em unidades públicas (estatais) ou privadas (não estatais), tendo em vista a integração cruzada do processo de oferta dos serviços, tal como preconizado nas ações de atendimento aos usuários/demandantes 
de tais serviços. Os modelos constitutivos da implantação/implementação na política de saúde universalizada no contexto brasileiro requereram a devida atenção dos executores na prática de racionalização procedimental nas unidades hospitalares. Tal processo é situação determinante para aplicação adequada de recursos limitados da política pública de saúde, considerando a crescente necessidade de uma população em transição.

A importância da prática de auditagem como mecanismo de racionalização das ações de controle interno beneficia a gestão de recursos em busca da otimização de procedimentos e práticas nas unidades hospitalares - a qual se conclui como monitoramento de custo/benefício. Nos procedimentos de auditoria externa, a questão fulcral é de transparência para a comunidade externa - seja órgãos fiscalizadores ou comunidade beneficiária, o que pode resultar na fase de accountability ou transparência, permitindo uma análise da viabilidade econômico-financeira da unidade hospitalar na prática gerencial da saúde.

Em síntese, as transformações na saúde brasileira, seja em unidades hospitalares públicas ou privadas, alteraram o procedimento gerencial, com destaque para racionalização das atividades das unidades hospitalares, elemento vinculante na administração de custos, via monitoramento e auditagem com finalidade clara no atendimento das necessidades dos operadores do sistema, garantindo a eficácia/eficiência na efetiva prestação do serviço de saúde.

\section{REFERÊNCIAIS}

BRASIL. Constituição da República Federativa do Brasil. Brasília: Senado Federal, 1988. Disponível em: <http://www.senado.gov.br/legislacao/const/con1988/CON1988_05.10.1988/CON1988.pdf>. Acesso em: 21 set. 2012.

BRASIL. LEI No 8.080 - de 19 de setembro de 1990 - Diário Oficial 20/9/90 - Lei orgânica da saúde Alterada, Brasília. DF, 19 set. 1999. Disponível em: <http://www.planalto.gov.br/ccivil_03/leis/18080. htm>. Acesso em: 21 set.2012.

BRASIL. Portaria Interministerial nº 1006/MEC/MS, Brasília. DF, Senado. 27 mai. 2004. Disponível em: <http://dtr2001.saude.gov.br/sas/PORTARIAS/Port2004/GM/GM-1006.htm>. Acesso em 21 set.2012.

BRASIL. Portaria n ${ }^{\circ}$ 254, de 27 de março de 2012, Brasilia. DF, SAS. Disponível em: <http://www. brasilsus.com.br/legislacoes/sas/1 12659-254.html>. Acesso em 26 set.2012

BRASIL. Resolução n ${ }^{\circ}$ 407/MS, Brasília. DF, Senado. De 11 de setembro de 2008. DOU n ${ }^{\circ}$ 4, de 11 mar. 2009. Disponível em: <http://www.saude.mt.gov.br/upload/noticia/2/arquivo/ 280710184043-SESMT-A-regimento-do-cns.pdf>. Acesso em: 21 set. 2012. 
BUSS, P. Problemas na saúde não se resolve apenas com dinheiro, diz diretor da Fiocruz. 2011. Disponível em: <http://www.jb.com.br/pais/noticias/2011/10/19/problemas-na-saude-nao-se-resolvem-apenascom-dinheiro-diz-diretor-da-fiocruz/>. Acesso em: 21 set. 2012.

CAMPOS, G. W.; BARROS, R. B.; CASTRO, A. M. Avaliação de política nacional de promoção da saúde. Brasília: Ministério da Saúde, 2004.

CARVALHO, A. I. de; BARBOSA, P. R. Políticas de saúde: fundamentos e diretrizes do SUS. Florianópolis: Departamento de Ciências da Administração - UFSC; Brasília: CAPES: UAB. 2010.

CASTRO, D. P. de. Auditoria e controle interno na administração pública. São Paulo: Atlas, 2008.

CFC - CONSELHO FEDERAL DE CONTABILIDADE. Princípios fundamentais de contabilidade e normas brasileiras de contabilidade. Brasília: CFC, 2001.

FELISBERTO, H. Da teoria à formulação de uma Política Nacional de Avaliação em Saúde: reabrindo o debate. 2006. Disponível em <http://www.atomicdog.com/trochim>. Acesso em: 26 set.2012.

GONÇALO, C. R; BORGES, M. de L. Organizações de saúde intensivas em conhecimento: um estudo no contexto de serviços de alta complexidade. Saúde e Sociedade, São Paulo, v.19, n.2, jun. 2010.

GURGEL Jr., G. D.; VIEIRA, M. M. F. Qualidade total e administração hospitalar: explorando disjunções conceituais. Ciência e Saúde Coletiva, São Paulo, v.7, n.2, 2002.

HOSPITAL DE CLÍNICAS. Dizendo sim à vida, ed. Especial 50 anos. 2011.

MAGAlhães, A. de D. F.; LUNKES; I. C.; MÜLlER, A. N. Auditoria das organizações: guia do estudante para a fundamentação do trabalho de pesquisa. São Paulo: Atlas, 2001.

MINISTÉRIO DA SAÚDE. Projeto Reforsus. Relatório final do estudo de novos modelos de gerência de unidades hospitalares. Por RIBEIRO, José Mendes e outros. Escola Nacional de Saúde Pública - ENSP/ Fiocruz, 2003.

RUA, M. das G. Políticas públicas. Florianópolis: Departamento de Ciências da Administração - UFSC; Brasília: CAPES: UAB. 2019. 
RUTHES, R. M.; CUNHA, I. C. K. O. Os desafios da administração hospitalar na atualidade. RAS, v. 9, n.36, p.93-102, jul./set. 2007.

SILVA, C. L. da; SOUZA-LIMA, J. E. de. Políticas públicas e indicadores para o desenvolvimento sustentável. São Paulo: Saraiva, 2010.

SOUZA, C. Políticas públicas: uma revisão da literatura. Revista Sociologias, Porto Alegre, ano8, n.16, p.20-45, jul./dez. 2006. 